\title{
Editorial
}

Respiration

Respiration 2006;73:145-146

DOI: $10.1159 / 000091530$

\section{Planned Admission to an ICU after Lung Resection: A Debatable Issue}

\author{
Alessandro Brunelli \\ Unit of Thoracic Surgery 'Umberto I' Regional Hospital, Ancona, Italy
}

The work of Pieretti et al. [1] published in this issue of Respiration evaluated an arbitrary - although clinically sound - protocol of planned admission to an ICU following major lung resection. They concluded that this protocol was effective in selecting those patients that could be safely admitted to the surgical ward without risk of major cardiorespiratory complications needing a late unplanned transfer to an ICU. The authors should be commended for their effort in raising interest in the important subject of postoperative intensive care management of lung resection patients. However, I found their results difficult to generalize and apply to other centers.

Often enough, the local health provider's organization is the primary factor dictating the policy of admission to an ICU. Some centers (as ours) send most of their patients to a dedicated general thoracic surgery ward with welltrained personnel and the availability of noninvasive continuous monitoring, reserving ICU mainly for patients likely to develop complications needing invasive assisted ventilation or invasive monitoring. Other institutions have access to an intermediate care unit or high dependency unit (HDU) [2]. By definition ICUs should be reserved for patients needing assisted ventilation; however, in the absence of a dedicated ward or an HDU, some centers (as that of Pieretti et al. [1]), 'over-admit' patients to ICUs. This policy not only causes inappropriate ICU bed occupation, increasing the hospital costs, but also delays patients' mobilization and increases the risk of complications, including, among others, infections, prolonged air leak and postoperative delirium.

Therefore, it appear logical to try and identify the predictors of an appropriate admission to an ICU in order to rationalize intensive care management. Unfortunately, the definition of a planned 'appropriate admission to an ICU' given by Pieretti et al. was arbitrarily founded on whether major complications occurred during the stay in the ICU stay and on the probability of needing an unplanned transfer to the ICU following care in the ward. In my view, this definition is questionable for at least two reasons: first, a selection bias appears evident, since the patients who were preselected and admitted to the ICU may have undergone a different treatment approach (i.e. a prolonged intubation period and a lower threshold for repeated bronchoscopies for sputum retention, which, incidentally, were both listed among the major complications) compared with those patients who were admitted to the ward. Second, the timing of complications, an important factor in my opinion, was not reported. Did the complications occur during the stay in the ICU or after the patients returned to the ward?

As a consequence, the ambiguous definition of the dependent variable renders the logistic regression results less reliable.

\section{KARGER}

Fax +4161306 1234

E-Mail karger@karger.ch

www.karger.com
(C) 2006 S. Karger AG, Basel

0025-7931/06/0732-0145\$23.50/0

Accessible online at: www.karger.com/res
Dr. Alessandro Brunelli

Via S. Margherita 23

IT-60129 Ancona (Italy)

Tel. +39071 596 4439, Fax +39071596 4433, E-Mail alexit_2000@yahoo.com 
To avoid a selection bias, a randomized study analyzing the outcome in terms of morbidity, mortality, length of hospital stay, and costs should be conducted, comparing similar patients who were and were not admitted to the ICU after lung resection.

In the absence of a randomization, balancing scores should be used to match patients and perform comparisons [3].

Another limitation of this study is the absence of ergometric data among the variables used for the analysis. As honestly admitted by the authors, exercise testing should now be an integral part of the preoperative evaluation and risk stratification processes.

Although the results generated by this work should be interpreted with caution and used in the clinical practice with even greater caution, I think they will open the way to future studies that will address important unanswered issues: the effect of management in an ICU on outcome compared with management in a surgical ward in com- parable groups of patients; the effect of management in an ICU on hospital costs; the prediction of admission to an ICU following initial recovery in lung resection patients.

Therefore, in my view, Pieretti et al. should be congratulated for having ventured into these still uncharted waters.
References
1 Pieretti P, Alifano M, Roche N, Vincenzi M, Forti Parri SN, Zackova M, Boaron M, Zanello M: Predictors of an appropriate admission to an ICU after a major pulmonary resection. Respiration 2006;73:157-165.

2 Klepetko W, Aberg THJ, Lerut AEMR, et al: Structure of general thoracic surgery in Europe: by The EACTS/ESTS Working Group on Structures in Thoracic Surgery. Eur J Cardiothorac Surg 2001;20:663-668.

3 Blackstone EH: Comparing apple and oranges. J Thorac Cardiovasc Surg 2002;123:8-15. 